\title{
T1 mapping in discrimination between hypertrophic and hypertensive cardiomyopathy
} \author{
Eike Nagel, Valentina Puntmann \\ From 17th Annual SCMR Scientific Sessions \\ New Orleans, LA, USA. 16-19 January 2014
}

Rocio Hinojar*, Benjamin P Goodman, Adriana Villa, Eduardo Arroyo Ucar, Darius Dabir, Tobias Schaeffter,

\section{Background}

The differential diagnosis of hypertrophic phenotype remains challenging in clinical practice, in particular between hypertrophy cardiomyopathy (HCM) and increased left ventricular wall thickness (LVWT) due to systemic hypertension. Diffuse myocardial fibrosis is the characteristic feature in HCM, whereas hypertensive response is underpinned by addition of myofibrils in otherwise normal myocardial tissue. Late gadolinium enhancement (LGE) imaging provided important new way of differentiation between these two entities by separating those cases with evidence of regional fibrosis. Whereas approximately $60 \%$ of patients with HCM reveal visually discernable LGE, T1 mapping is highly discriminative, irrespective of the presence of LGE.

\section{Methods}

Sixty patients with diagnosis of unequivocally hypertrophic cardiomyopathy and fifty patients with hypertensive cardiomyopathy underwent routine cardiac MRI protocol including assessment of function and scar (3-Tesla). $\mathrm{T} 1$ values were measured conservatively within septal myocardium in midventricular short-axis slice prior to administration of $0.2 \mathrm{mmol} / \mathrm{kg}$ of gadobutrol.

\section{Results}

HCM group showed higher LV mass and maximum LVWT than the hypertensive group (HCM vs. hypertensive: LVmass, g/m2: $97 \pm 31$ vs. $69 \pm 22$; maximum LVWT $18.4 \pm 3$ vs. $13.3 \pm 1.4, \mathrm{p}<0.0001)$. There was LGE in $20 \%$ of hypertensive $(\mathrm{n}=10,4$ of them with an ischaemic pattern) and in $84 \%$ of hypertrophic cardiomyopathy $(\mathrm{n}=50,3$ of them with an ischaemic pattern) $(\mathrm{p}<0.001)$. Patients with HCM showed significantly

Cardiovascular Imaging Department, King's College London, London, UK, London, UK
We demonstrate that native $\mathrm{T} 1$ values can reliably discriminate between hypertrophic and hypertensive

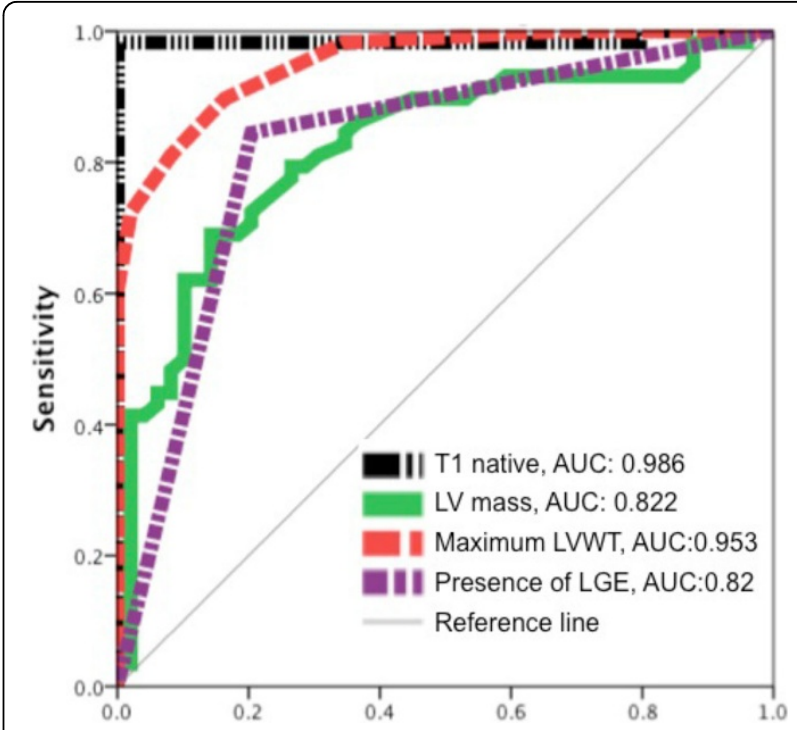

Figure 1 Diagnostic Performance of T1 Mapping compared with LV mass, maximum LVWT and the presence of LGE in the differentiation between HCM and hypertensive cardiomyopathy. 
cardiomyopathy. We propose that native $\mathrm{T} 1$ may serve as a novel diagnostic marker to discriminate between the two hypertrophic conditions.

\section{Funding}

We would like to acknowledge Department of Health via the National Institute for Health Research (NIHR) comprehensive Biomedical Research Centre award to Guy's \& St Thomas' NHS Foundation Trust in partnership with King's College London and King's College Hospital National Health Service Foundation Trust. Dr. Rocio Hinojar was supported by the Fundacion Alfonso Martin Escudero.

Published: 16 January 2014

- Convenient online submission

- Thorough peer review

- No space constraints or color figure charges

- Immediate publication on acceptance

- Inclusion in PubMed, CAS, Scopus and Google Scholar

- Research which is freely available for redistribution 